\title{
Chemotherapy Induced Erythroid Dysplasia in a Patient with Acute Myeloid Leukemia
}

Mohammad Faizan Zahid $^{1 *}$, Siddhart Bhattacharyya ${ }^{2}$ and Irma E Palazzo ${ }^{3}$

${ }^{1}$ Department of Medical Oncology, Fox Chase Cancer Center, Philadelphia, USA

${ }^{2}$ Department of Pathology and Laboratory Medicine, Temple University Hospital, USA

${ }^{3}$ Department of Pathology and Laboratory Medicine, Jeanes Hospital, USA

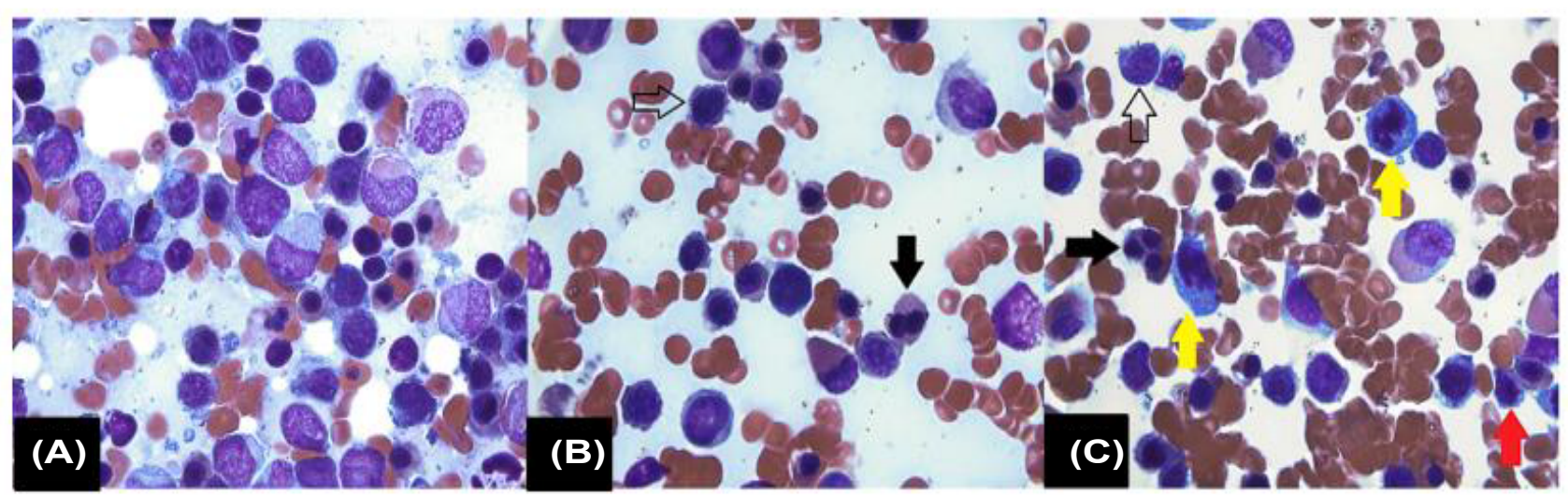

A 23 years old gentleman presented with a reduced appetite, weight loss and fatigue. $\mathrm{CBC}$ revealed anemia, thrombocytopenia and elevated WBC count with $75 \%$ blasts on peripheral blood. Bone marrow aspirate and biopsy showed hypercellular marrow with $60 \%$ involvement by myeloblasts and no dysplastic changes (Panel A). Flow cytometry identified a distinct blast population (68.8\%) expressing myeloblastic markers. Cytogenetics showed no chromosomal abnormalities. The diagnosis was consistent with acute myeloid leukemia (AML) and the patient was started on chemotherapy. On day 22 he spiked fever. CBC showed persistent pancytopenia. His bone marrow biopsy showed a normocellular marrow with $25 \%$ involvement by myeloblasts. Dysplastic erythroid precursors were seen in the background. Morphologic features including nuclear budding (red arrows), karyorrhexis(yellow arrows), multinucleation (black arrows) and megaloblastoid changes(transparent arrow) (Panels B\&C) were observed. Flow cytometry confirmed a distinct population of blasts (21\%) with similar immunophenotype as previously, consistent with residual AML. This case highlights a dramatic change in erythroid precursor morphology highly suggestive of dysplasia occurring after chemotherapy. Though initial cytogenetic studies did not yield any evidence of underlying myelodysplasia, this method may not have detected minimal chromosomal errors like micro-deletions, which could explain the abrupt emergence of dysplastic features in normal bone marrow precursors. Additional studies, such as next generation DNA sequencing and chromosomal microarray analysis can potentially detect such minor chromosomal errors that would otherwise remain undiagnosed by conventional cytogenetics. We did not perform these additional tests at initial presentation. However, we conclude, in this clinical context and with the short time frame, that the dyspoietic changes are most likely secondary to chemotherapy effect.

*Corresponding author: Mohammad Faizan Zahid, Department of Medical Oncology, Fox Chase Cancer Center, 333 Cottman Avenue, Philadelphia, PA 19111, USA, Tel: +1 516728 0490; E-mail: faizanzahid91@hotmail.com

Received September 05, 2014; Accepted September 24, 2014; Published September 29, 2014

Citation: Zahid MF, Bhattacharyya S, Palazzo IE (2014) Chemotherapy Induced Erythroid Dysplasia in a Patient with Acute Myeloid Leukemia. J Blood Disorders Transf 5: 230. doi: 10.4172/2155-9864.1000230

Copyright: @ 2014 Zahid MF, et al. This is an open-access article distributed under the terms of the Creative Commons Attribution License, which permits unrestricted use, distribution, and reproduction in any medium, provided the original author and source are credited. 